\title{
Respuesta con enfoque de derechos de la niñez frente a la pandemia por COVID-19 en Chile, Colombia y Perú
}

\author{
Fernando González, ${ }^{1}$ María Camila Pinzón-Segura, ${ }^{2}$ Bertha Luz Pineda-Restrepo, ${ }^{3}$ María del \\ Carmen Calle-Dávila, ${ }^{4}$ Estefanía Siles Valenzuela, ${ }^{5}$ Natalia Herrera-Olano ${ }^{6}$ y María Lucía Mesa Rubio ${ }^{7}$
}

Forma de citar

González F, Pinzón-Segura MC, Pineda-Restrepo BL, Calle-Dávila MC, Siles Valenzuela E, Herrera-Olano N et al. Respuesta con enfoque de derechos de la niñez frente a la pandemia por COVID-19 en Chile, Colombia y Perú. Rev Panam Salud Publica. 2021;45:e151. https://doi.org/10.26633/RPSP.2021.151

RESUMEN

Objetivo. Describir las estrategias que fueron establecidas por Chile, Colombia y Perú durante el primer año de la pandemia por COVID-19 y compararlas desde el enfoque de derechos de la niñez.

Métodos. Se realizó un estudio cualitativo de análisis comparado de políticas públicas, tomando como eje siete categorías construidas por el Capítulo Latinoamericano de la International-Society-for-SocialPediatrics-and-Child-Health a partir de la Convención de Derechos de la Niñez (CDN). La selección de los documentos de los países se realizó por conveniencia y su análisis en diálogos deliberativos.

Resultados. Se revisaron 173 documentos de los tres países. Destaca como convergencia la priorización de la prevención de la transmisión comunitaria del virus, por sobre la promoción del ejercicio de derechos de la niñez, la falta de participación de niños, niñas y adolescentes (NNA) en el proceso de elaboración de las políticas públicas, y la falta de avance en el reconocimiento y protección del ejercicio de todos sus derechos. No hubo mayores divergencias más allá de brechas de desigualdad identificadas con base a la realidad de cada país.

Conclusión. La pandemia ha afectado el funcionamiento de los sistemas económicos, sociales, de salud, educación, medioambiente y gobernanza de estos tres países. Si bien este estudio muestra un avance en la inclusión del enfoque de derechos de NNA en las políticas formuladas, su comprensión como sujetos sociales y políticos titulares de derecho podría permitir la construcción de alternativas colectivas que garanticen la salud y el bienestar para todas las personas en el curso de vida.

Palabras clave COVID-19; defensa del niño, política pública; cuidado del niño; salud del niño; Chile; Colombia, Perú.

Latinoamérica y el Caribe (LAC) ha sido una de las regiones del mundo más afectadas por el coronavirus (COVID-19), representado en el número de casos como de muertes (1). En este contexto, la región enfrenta el complejo desafío de controlar la pandemia sin dejar de garantizar el ejercicio de derechos de su población. La propagación del COVID-19 y sus consecuencias se ven agravados por problemas estructurales internos de los países de la región, como son los elevados niveles de desigualdad, informalidad laboral, pobreza, vulnerabilidad y asentamientos urbanos marginados, así como sus sistemas de salud, educación y de protección social débiles y fragmentados (2-6).

\footnotetext{
Oficial de Salud UNICEF Chile, Santiago de Chile. $\triangle$ Fernando González, fegonzalez@unicef.org

2 Facultad de Derecho y Ciencia Política de la Universidad Nacional de Colombia, Bogotá, Colombia.

3 Organismo Andino de Salud - Convenio Hipólito Unanue, Lima, Perú.
}

\footnotetext{
4 Organismo Andino de Salud - Convenio Hipólito Unanue, Lima, Perú.

5 Hospital Luis Calvo Mackenna, Santiago de Chile, Chile.

6 Universidad de los Andes, Bogotá, Colombia.

7 Asesora Despachó del Ministro de Salud y Protección Social, Bogotá, Colombia.
}

Este es un artículo de acceso abierto distribuido bajo los términos de la licencia Creative Commons Attribution-NonCommercial-NoDerivs 3.0 IGO, que permite su uso, distribución y reproducción en cualquier medio, siempre que el trabajo original se cite de la manera adecuada. No se permiten modificaciones a los artículos ni su uso comercial. Al reproducir un artículo no debe haber ningún indicio de que la OPS o el artículo avalan a una organización o un producto específico. El uso del logo de la OPS no está permitido. Esta leyenda debe conservarse, junto con la URL original del artículo. Crédito del logo y texto open access: PLoS, bajo licencia Creative Commons Attribution-Share Alike 3.0 Unported. 
El escaso reconocimiento de niños, niñas y adolescentes (NNA) como sujetos de derechos en las políticas públicas ha dificultado el ejercicio de los derechos consagrados en la Convención de Derechos de la Niñez (CDN). La pandemia y las medidas tomadas por los gobiernos para su control han amplificado aún más esta brecha en función de proteger la salud de la población. Por esto se hace relevante comprender el impacto de la COVID-19 en NNA en la región, considerando un enfoque de derechos de la niñez, entendido como un marco conceptual normativo y operacional -basado en la CDN-, que brinda orientaciones para la promoción y la protección de los derechos en propio articulado, así como mediante las observaciones generales del comité de derechos del niño de la Organización de Naciones Unidas (7-10).

En este esfuerzo, destacan los procesos investigativos generados por la Sociedad Internacional de Pediatría Social (International-Society-for-Social Pediatrics-and-Child-Health, ISSOP), que permitieron el intercambio de experiencias y reflexiones desde el comienzo de la pandemia hasta la fecha. Si bien este espacio identificó que los gobiernos, la academia y diversas organizaciones no gubernamentales (ONGs) estaban desarrollando estrategias y acciones para prevenir y dar respuesta a los impactos de la COVID-19 en NNA, no existía hasta la fecha un análisis para entender su impacto en términos del ejercicio de derechos para este grupo. El objetivo de este artículo es describir y comparar las estrategias establecidas por tres países de la región durante el primer año de la pandemia, desde un enfoque de derechos de la niñez, a fin de identificar lecciones a futuro para los países de la Región de las Américas.

\section{MATERIALES Y MÉTODOS}

Se trata de un estudio cualitativo descriptivo de análisis comparado de políticas públicas, (11-13) que contó con una fase de adaptación de la metodología de diálogos deliberativos y otra de análisis de contenido, orientadas por la pregunta: ¿Cuáles son los elementos centrales de la formulación de políticas públicas, con enfoque de derechos de la niñez y adolescencia, en tiempos de pandemia COVID-19?

\section{Selección de países, estrategia de búsqueda y selección de documentos}

Los espacios de diálogo generados por el capítulo de LAC de la ISSOP permitieron intercambios que promovieron la realización de esta investigación. En estas reuniones virtuales de trabajo se presentaba la situación de cada país participante y el impacto que se estaba evidenciando particularmente sobre la salud de NNA. Inicialmente participaron representantes de ocho países de la región quienes aportaron en el análisis de la situación general. Posteriormente, y de acuerdo al interés y disponibilidad de los participantes, Chile, Colombia y Perú se mantuvieron en las sesiones de deliberación con dos investigadores que contaban con capacidad de articular los puntos de vista y las experiencias de los grupos que representaban.

Con el propósito de comprender el sentido del discurso de las estrategias de mitigación de los efectos de la pandemia sobre NNA, y privilegiando el lenguaje escrito (texto) como instrumento para comprender significantes de los tomadores de decisión, se realizó una búsqueda de documentos prescriptivos, en sitios web de ministerios, autoridades sanitarias, así como de sociedades científicas o académicas reconocidas en Chile, Colombia y Perú (14-16).

Se consideraron criterios de inclusión: 1) haber sido publicados entre 2020 y marzo del 2021; 2) que reflejaran recomendaciones de tipo prescriptivo para mitigar los efectos de la pandemia por COVID-19 sobre NNA; y 3) que hayan sido escritos bajo el marco de enfoque de derechos humanos. Como único criterio de exclusión se consideró la imposibilidad de acceder al contenido del documento. Los documentos fueron clasificados con base a su carácter y procedencia en: a) gubernamental: normativo y regulatorio; b) promoción, educación y/o difusión de información; c) recomendaciones de la académica, asociación científica o tercer sector; d) documentos con alcance regional.

Los documentos fueron ingresados a una planilla, recopilando nombre, alcance, resumen, número de páginas, enlace de acceso al texto completo, fecha de publicación e inclusión de alguno de los ejes de comparación prestablecidos.

\section{Diálogos Deliberativos}

La metodología de diálogos deliberativo se definen como: "mecanismo interactivo para compartir conocimientos [cuyo] objetivo es respaldar la discusión completa de las consideraciones relevantes sobre un asunto de alta prioridad, con el fin de informar la toma de decisiones en políticas y otro tipo de acción [así como] proveer oportunidades para la discusión del problema identificado, las opciones para abordarlo y temas relacionados con barreras, facilitadores o puntos a tener en cuenta respecto a la implementación de aquellas opciones" (13). Los encuentros se llevaron a cabo entre los países vinculados al capítulo LACISSOP, entre marzo de 2020 y mayo de 2021, conducido por una facilitadora capacitada quien garantizó que la discusión se mantuviera centrada en los asuntos primordiales y que se escuchasen todas las voces desde una postura crítica. Los pasos llevados a cabo en los 15 diálogos se presentan en el recuadro 1.

\section{Análisis de contenido}

Partiendo de la hipótesis de trabajo derivada de los primeros encuentros: "las políticas públicas para mitigar los efectos de la COVID en NNA de la región se encuadran en un discurso adulto-céntrico" se buscaron, a través del análisis de contenido de los documentos, las ideas expresadas, siendo las unidades de observación el significado de las frases y párrafos, relacionados con la temática (17).

Se comenzó con la lectura de la CDN, a partir de la cuál, en sesión de diálogos, se construyeron siete categorías sobre derechos de la niñez relacionadas con el contexto de la pandemia, presentadas en el recuadro 2.

Una vez definidas las categorías, se realizó a un ejercicio de codificación de base gramatical, llevada a cabo por los investigadores y dirimido por la facilitadora, a fin de confirmar que las categorías fueran homogéneas, exhaustivas, exclusivas, objetivas y adecuadas o pertinentes. Posteriormente, cada uno de los investigadores realizó la codificación de los textos seleccionados por su país, y en caso de diferencias a la hora de comparar codificaciones realizadas, la facilitadora dirimía las mismas. Finalmente, se organizaron los datos en tablas, a partir de las cuales se realizaron inferencias y comparaciones, durante subsecuentes diálogos deliberativos, con el grupo completo de investigadores. 
RECUADRO 1. Pasos de los Diálogos Deliberativos para la recolección de documentos y análisis de la respuesta con enfoque de derechos de la niñez frente a la pandemia.

- Envío por parte de la facilitadora, previo a la reunión, del resumen de la temática, aspecto metodológico o política a analizar

- En el caso de temática o aspecto metodológico a tratar: exposición por parte de la facilitadora de argumentos de discusión; en el caso de política a analizar: exposición por parte del país del que derivó el documento de características del problema, afectación a NNA y sus familias, alternativas de abordaje y consideraciones clave de implementación

- Defensa 0 argumentación de los aspectos clave a incluir o comparar por parte del expositor

- Deliberación abierta al resto de investigadores, con espacio para contra argumentar

- Levantamiento de la información a través de resúmenes con disensos o consensos de las discusiones derivadas de cada documento de política Fuente: elaboración propia

RECUADRO 2. Categorías construidas sobre los derechos de la niñez afectados por la pandemia de COVID-19.

1. Estrategias para disminuir o mitigar el impacto sobre la salud física y mental de NNA causadas por las medidas de control de transmisión del COVID-19.

2. Estrategias para identificar y abordar el riesgo psicosocial.

3. Estrategias para evitar la interrupción de cuidados y procesos terapéuticos.

4. Promoción del acompañamiento de las gestantes y de NNA durante el parto y/ u hospitalización

5. Protocolos diferenciados para NNA en situación de marginalidad

6. Participación de NNA

7. Estrategias que aseguren bienes básicos a familias con NNA

Fuente: elaboración propia a partir de las categorías construidas

\section{RESULTADOS}

Los tres países difieren en las curvas de contagios, sin embargo, tienen similitudes en las tasas de incidencia de casos COVID-19 en la población general y en NNA, así como la tasa de fallecidos, información relevante para la interpretación de los resultados (cuadro 1).

Se revisaron 173 documentos, 56 de Chile, 58 de Colombia y 59 de Perú, 121 tenían carácter normativo o regulatorio (69.9\%); 38 educativos y de difusión de información (19.0\%); 15 derivados de asociaciones científicas (8.6\%), y 4 de alcance regional, siendo escritos o editados por agencias de las Naciones Unidas (cuadro 2).

A continuación, se presenta una síntesis de la revisión documental categorizada, junto con un análisis conjunto de las estrategias.

\section{Estrategias para disminuir o mitigar el impacto sobre la salud física y mental de NNA}

Los tres países desarrollaron tempranamente estrategias para reducir el contagio de COVID-19, y en distintos tiempos medidas para mitigar sus impactos sobre la salud de NNA. Colombia, a dos meses de iniciada la pandemia, presentó una estrategia para el cuidado de la salud de NNA, con recomendaciones para brindar acompañamiento socioemocional en casa. Priorizaron a NNA con condiciones de salud mental en desescalonamiento del aislamiento. En Chile, al cuarto mes de iniciada la pandemia se lanza el plan "paso a paso", con medidas y excepciones para NNA del espectro autista y trastornos conductuales, y se elaboró la Guía Práctica de Bienestar Emocional y la plataforma SaludableMente para apoyar su salud mental y el bienestar psicosocial. Perú estableció excepciones al desplazamiento de NNA fuera del domicilio en tiempos de confinamiento al segundo mes de la pandemia.
Se elaboró la Guía-técnica-para-cuidado-de-la-salud-mental con capítulos específicos de NNA, y el Plan de Salud Mental en el contexto COVID-19.

Los tres países establecieron medidas de confinamiento y restricción de desplazamiento como cierre de escuelas y espacios públicos. Sin embargo, dispusieron franjas horarias en las cuales se podía salir a espacios abiertos, durante la cuarentena. Los tres países desarrollaron estrategias de apoyo socioemocional de NNA y sus familias y dispusieron, en distintos tiempos, canales informativos para la prevención de la enfermedad.

\section{Estrategias para identificar y abordar riesgo psicosocial}

Los tres países establecieron acciones para prevenir y denunciar el maltrato infantil. En Chile, se crearon canales de comunicación específicos (FonoNiños-147 de Carabineros de Chile para NNA víctimas de vulneraciones de derechos; FonoFamilia-149 para orientación y auxilio a familias; FonoInfancia para apoyo psicológico de la crianza; y LíneaLibre-1515 para brindar orientación psicológica a NNA desde ONGs). Se implementó la campaña ciudadana "Mascarilla-19" para atender a mujeres víctimas de violencia intrafamiliar. En Colombia, se publicaron orientaciones para prevenir las violencias contra NNA ofreciendo redes de apoyo (REDPAPAZ) y acceso a información institucional virtual. Se publicaron lineamientos para la atención de salud de NNA con COVID-19, con apartados para la prevención y detección de riesgo psicosocial estableciendo signos de alarma para su atención inmediata e integral. En Perú, el Ministerio de la Mujer y Poblaciones Vulnerables, fortalecieron canales de denuncia frente a situaciones de riesgo o desprotección familiar de NNA y establecieron mecanismos para garantizar el ejercicio de sus derechos. Se aprobó el Reglamento de Mecanismos de Prevención de Violencia en la Familia, 
CUADRO 1. Indicadores demográficos y epidemiológicos relacionados con la COVID-19 en Chile, Colombia y Perú

\begin{tabular}{|c|c|c|c|}
\hline País & Chile & Colombia & Perú \\
\hline Población & 18,7 millones de habitantes & 51.2 millones de habitantes & 34,1 millones de habitantes \\
\hline Fecha del Primer caso COVID-19 & 3 de marzo, 2020 & 6 de marzo, 2020 & 6 de marzo, 2020 \\
\hline Número de contagios* & 864000 & 2273245 & 1371176 \\
\hline Número de contagios en menores de 19 años* & 69500 & 170201 & 110197 \\
\hline Casos confirmados por 100000 habitantes* & 4500 & 4440 & 4019 \\
\hline $\begin{array}{l}\text { Casos confirmados por } 100000 \text { habitantes menores de } \\
19 \text { años }^{*}\end{array}$ & 1800 & 1072 & 978 \\
\hline Número de fallecidos* & $28686^{* *}$ & 60412 & 47854 \\
\hline Número de fallecidos en menores de 19 años* & $265^{\star *}$ & 306 & 287 \\
\hline Fallecidos por 100.000 habitantes* & 110 & 118 & 140 \\
\hline
\end{tabular}

* a 1 año del primer caso; ** Confirmados + sospechosos;

Fuente: elaboración propia a partir de las fuentes oficiales de cada país reportadas por los Ministerios de Salud.

CUADRO 2. Características de los documentos revisados por país.

\begin{tabular}{|c|c|c|c|c|}
\hline & Chile & Colombia & Perú & Total \\
\hline Totalidad de documentos aportados Número (\%) & $\begin{array}{c}56 \\
(32,3 \%)\end{array}$ & $\begin{array}{c}58 \\
(33,5 \%)\end{array}$ & $\begin{array}{c}59 \\
(34,1 \%)\end{array}$ & 173 \\
\hline Documento nacional con carácter normativo y regulatorio. & 40 & 41 & 40 & 121 \\
\hline Documentos de promoción, educación y difusión de información. & 11 & 8 & 40 & 33 \\
\hline Recomendaciones de la academia, asociación científica o tercer sector. & 5 & 6 & 4 & 15 \\
\hline Documentos con alcance regional: UNICEF, OIM, OPS/OMS. & 0 & 3 & 1 & 4 \\
\hline
\end{tabular}

Fuente: elaboración propia a partir de los resultados obtenidos

y se publicaron los Protocolos para la Gestión de la Convivencia Escolar la Prevención y Atención de la Violencia contra NNA. Además, la Defensoría del Pueblo elaboró informes especiales sobre la materia.

En los tres países se crearon canales de comunicación y respuesta para la denuncia de problemas de riesgo psicosocial, así como redes de apoyo. Sobre este punto en los diálogos deliberativos hubo convergencia, en que existe un silencio respecto a sistemas de monitoreo activo a las violencias (que se han visto incrementadas durante la pandemia) que demuestren la protección efectiva de los casos identificados.

\section{Estrategias para evitar la interrupción de cuidados y procesos terapéuticos}

En los tres países se establecen estrategias para evitar la interrupción de cuidados y procesos terapéuticos, priorizando los más críticos. Hay convergencia en la priorización del control presencial de gestantes, recién nacidos y lactantes, programas de inmunizaciones, y continuidad a la atención en planificación familiar. En Chile se publicó el protocolo para prevención de transmisión de COVID-19 en unidades de pediatría y de cuidados intensivos, promoviendo la hospitalización acompañada. Perú publicó un decreto supremo para garantizar las prestaciones de prevención y control de la anemia; normas referentes al Plan de Recuperación de Brechas en Inmunizaciones y Anemia y medidas para incrementar su capacidad de respuesta de autoridades locales de salud. En relación al manejo del COVID-19 y sus presentaciones graves, los tres países desarrollaron protocolos de atención, así como protocolos para el manejo del síndrome inflamatorio multisistémico pediátrico.
En los diálogos deliberativos hay concordancia sobre la necesidad de evaluar el funcionamiento de dichas estrategias y su efectividad en la mitigación efectiva del impacto de la COVID-19 y la pandemia sobre la salud de NNA.

\section{Promoción del acompañamiento de las gestantes durante el parto y de la hospitalización de NNA}

Todos los países establecieron protocolos de acompañamiento en el parto, pero en distintos tiempos. En Chile, se establecieron protocolos de acompañamiento al quinto mes del primer caso. Al sexto mes se publicó la orientación técnica que recomienda el acompañamiento de NNA en casos específicos como lactantes y NNA con trastornos de la conducta o del espectro autista. En Colombia, se elaboró el protocolo de acompañamiento en el parto, al tercer mes del primer caso de COVID-19 y en el Perú, se promulgó el protocolo de acompañamiento en el parto, al cumplir un mes del primer caso. Sobre el acompañamiento de NNA hospitalizados, hay menos información. Todos los protocolos establecen normativas para garantizar un acompañamiento seguro para todos los involucrados, y para gestionar adaptaciones al espacio físico según la realidad local.

\section{Protocolos diferenciados para NNA en situación de marginalidad}

En Chile, el Subsistema de Protección Integral de la Infancia Chile Crece Contigo, estableció estrategias para acercar los servicios sociales a la comunidad. Los Ministerios de Salud, Educación y Desarrollo Social y Familia (MDSF) elaboraron material en los idiomas de pueblos originarios e inmigrantes. El Servicio 
Nacional de Discapacidad, con agrupaciones de la sociedad civil, pacientes y academia, elaboraron materiales que brindan información a personas con discapacidad sobre sus derechos, y recomendaciones a los equipos de salud para brindar atención segura a estas poblaciones. El MDSF también estableció estrategias para disminuir el riesgo de contagio en población migrantes y familias en situación de pobreza. En Colombia, se elaboraron protocolos para facilitar la identificación de NNA en el Sistema de Responsabilidad Penal Adolescente (SRPA) y a aquellos desvinculados del conflicto armado, y su afiliación al sistema de seguridad social. Se dispusieron lineamientos para la prevención, contención y mitigación de COVID-19 y atención de salud en adolescentes del SRPA y migrantes. En el Perú, se aprobó el decreto que establece acciones para la protección de los pueblos originarios, con atención prioritaria a NNA; también se establecieron lineamientos para identificar casos sospechosos de COVID-19 en pueblos indígenas y afroperuanos para realizar seguimiento y monitoreo durante el tratamiento. Fue elaborado material educativo en lenguas originarias y se crearon normas para enmendar el hacinamiento en establecimientos penitenciarios con medidas socioeducativas para adolescentes privados de libertad. Se entregaron tabletas con internet a instituciones educativas públicas con prioridad en alumnos en situación de pobreza. Se brindó atención alimentaria complementaria y se aprobaron los modelos de "Atención Oportuna de NNA en presunto estado de abandono".

Los tres países presentaron estrategias focalizadas para mejorar el acceso a servicios sociales y de salud de la población y de NNA en situación de marginalidad (discapacidad, migración, grupos étnicos, entre otros).

\section{Participación de NNA}

En Chile, el Ministerio de Salud abrió espacios de diálogo con NNA para reflexionar sobre el cierre de las escuelas y el impacto de la restricción de desplazamiento. Si bien se recogió sistemáticamente su opinión, no queda claro si fueron considerados en la toma de decisiones por parte de la autoridad. La Sociedad Chilena de Pediatría y la Defensoría de la Niñez generaron espacios de participación y publicaron información levantada para movilizar a los tomadores de decisión a favor de la niñez. En el Perú, se han mantenido activos espacios de participación de NNA como el Colectivo Interinstitucional por los Derechos de la Niñez y Adolescencia, el Consejo Consultivo de NNA, y la Mesa de Concertación para la Lucha Contra la Pobreza. Se han realizado foros y conversatorios intergeneracionales sobre protección de derechos en el contexto de COVID-19 por diversas organizaciones. Se destaca también el Organismo Andino de Salud - Convenio Hipólito Unanue con actividades con NNA para promover el autocuidado y elaborar la sistematización que llevó por título: "¿Qué dicen los NNA sobre la vacunación contra COVID19 en Perú?" Aunque la participación es un asunto prioritario en el marco normativo general de Colombia, en la revisión documental no se reportaron estrategias de políticas actualizadas de participación infantil en el marco de la COVID-19.

\section{Estrategias que aseguren bienes básicos}

Esta es la categoría con más divergencias, principalmente por las problemáticas particulares de cada país. El elemento transversal fueron los apoyos vía transferencias directas o subsidios de distintos montos y a distintos tiempos. En cuanto a seguridad alimentaria de NNA, en Colombia, se dictaron medidas que brindan herramientas a las entidades territoriales para garantizar la ejecución del Programa de Alimentación Escolar (PAE) a todas las instituciones educativas públicas del país. En Perú se entregaron canastas de alimentos para más de 2,5 millones de hogares, al igual que en Chile en que el alimento que se daba en las escuelas se entregó como canastas familiares.

En los tres países se establecieron disposiciones para fraccionamiento de pago de servicios básicos, se adelantaron las transferencias de programas sociales y hubo provisión excepcional de alimentos para población en situación de pobreza. En Perú se brindó asistencia económica para NNA cuyos cuidadores hayan fallecido por COVID-19. En Chile, tras importantes demandas ciudadanas, el poder judicial sentenció al Estado para que asegurara 100 litros de agua potable por día por persona y el apoyo económico ha sido por medio de tres mecanismos: retiros de los propios ahorros de los fondos de pensiones; la entrega de un ingreso familiar de emergencia al $60 \%$ de las familias con mayor necesidad económica del país; y un préstamo estatal solidario a los individuos que lo solicitaron.

En los tres países se evidenciaron brechas de acceso a servicios básicos, saneamiento, emergencia de trabajo informal, trabajo infantil entre otros problemas sociales, a los que se han dado respuestas coyunturales, principalmente por políticas focalizadas.

\section{DISCUSIÓN}

La investigación identifica y contrasta elementos presentes o ausentes en la formulación de las políticas públicas establecidas para enfrentar la pandemia, desde una perspectiva de derechos de la niñez. Corresponde señalar como limitación la selección de países participantes por conveniencia, lo que no permite tener una visión amplia y acabada de lo que ocurre en la región. Respecto a la estrategia de búsqueda, si bien no se basó en una revisión sistemática, ésta cumplió con chequeo de pares y verificación externa. A su vez es necesario destacar que, dado el curso incierto de la pandemia, toda conclusión es preliminar y parcial. Teniendo en cuenta que este estudio analiza las bases discursivas de las políticas públicas formuladas, se consideraría adecuado un nuevo análisis comparativo, en caso de que se observe un giro en base epistemológica de los enfoques de las políticas públicas.

La pandemia por COVID-19, así como la respuesta establecida por los gobiernos han interferido ampliamente con las actividades humanas generando amplios efectos en los sistemas de políticos, económicos y sociales, en todo el mundo (18). Los resultados presentados en este estudio, al igual que publicaciones de otras regiones del mundo sugieren que el reconocimiento y protección de los derechos de la CDN se han visto importantemente restringidos (19). Algunos derechos intentaron ser restituidos por vías alternativas con resultados parciales como el aumento de las brechas de trayectorias educativas por nivel socioeconómico (20), género (21) y la conectividad (22), como consecuencia de la impartición de educación virtual en diversos países de la región (23). Si bien esta dimensión no fue profundizada en este estudio, apareció transversalmente en todas las categorías.

El aumento de las brechas sociales y la exacerbación de situaciones de vulnerabilidad se tradujeron en la elevación 
de contagios y letalidad en poblaciones específicas. El acompañamiento a dichas familias, la continuidad de los servicios y el fortalecimiento de la protección social fueron claves para el desarrollo de la resiliencia comunitaria (24). Considerando la inequidad de la región, se recomienda que los gobiernos implementen medidas efectivas para disminuir el contagio y tratar la enfermedad, sin descuidar el proceso salud-enfermedad, impulsando modelos de cuidado y atención a las familias de base comunitaria, así como su participación en la toma de decisiones (25).

Respecto al derecho a ser oídos, Perú y Chile sobresalieron, ya que, a través de consejos consultivos de NNA, han contribuido al avance hacia formas de participación auténtica, superando los tres primeros peldaños -manipulación, decoración y participación simbólica- de la escala de Hart (26). Aunque Colombia no reportó estrategias actualizadas en respuesta a la COVID-19, éste destaca por sus políticas de participación social en salud (27).

En relación al análisis discursivo, la principal convergencia muestra que, a pesar del temprano consenso científico respecto al rol menor de los NNA en la propagación del virus, las estrategias se focalizaron en restricciones de desplazamiento y prolongados cierres de espacios educativos y recreativos fundamentales para el desarrollo de NNA, con sus deletéreas consecuencias $(28,29)$. En diálogo con estudios que afirman que las políticas establecidas y sus modos hegemónicos de formulación no son circunstanciales, sino estructurales (30), nuestros resultados sugieren que las políticas públicas analizadas están enmarcadas en enfoques convencionales, los cuales buscan resolver problemas desde comprensiones adultocéntricas, lineales, técnico científicas y con escasa participación de los sujetos a quienes van dirigidas, reproduciendo así las inequidades del modelo civilizatorio epocal (31). Al respecto, el análisis muestra la necesidad de que los países consideren a los NNA como sujetos sociales y políticos, e innoven en maneras alternativas para su participación en los asuntos que les competen $(25,32)$. Los enfoques interpretativo-críticos de análisis de políticas públicas, la medicina social y la salud colectiva (33), con teorías latinoamericanas específicas como la epidemiología crítica y la determinación social en salud podrían ser referentes para avanzar en nuevas formas de elaborar políticas públicas en salud (30,32-35).
En conclusión, el análisis confirma la necesidad de que los países consideren a los NNA como sujetos de derecho en el ciclo de las políticas públicas. Con la pandemia por COVID-19, ha quedado demostrado que tanto las características de los países como los enfoques imperantes de análisis de políticas públicas en salud requieren poner especial atención a la participación deliberativa de los NNA. Los resultados también pueden ser útiles para otros países de la región o del mundo, para el enfrentamiento de otras situaciones en donde los derechos entren en conflicto. El ejemplo del enfrentamiento de la pandemia por VIH/Sida y su tránsito desde un abordaje biomédico asistencial a uno biopsicosocial con enfoque de derechos, podría servir como referente en la coyuntura sanitaria y social actual, particularmente desde una mirada de los derechos de la niñez. Abordar los efectos sociales a largo plazo de la pandemia, incluido el deterioro de la salud mental, las inequidades y la pobreza, serán temas centrales para la resiliencia social en la fase de recuperación.

Contribución de los autores. Todos los autores concibieron el estudio original, participaron en la construcción metodológica, recolectaron los documentos, los analizaron e interpretaron los resultados y participaron tanto en la escritura como en la corrección del manuscrito. Todos los autores revisaron y aprobaron la versión final del mismo.

Agradecimientos. Agradecemos al grupo de ISSOP Latinoamérica por el constante apoyo, respaldo y confianza en el desarrollo del estudio. Particularmente a los doctores Ernesto Durán, Raúl Mercer e Iván Silva, quienes participaron como revisores externos y validadores de las discusiones recogidas en los diálogos deliberativos, lo que permitió llegar a los resultados presentados y enriquecer las conclusiones y discusiones del estudio; y a Pablo Villalobos Dintrans quien nos apoyó en revisión final de estilo, edición y redacción del manuscrito.

\section{Conflicto de intereses. Ninguno declarado por los autores.}

Declaración. Las opiniones expresadas en este manuscrito son responsabilidad del autor y no reflejan necesariamente los criterios ni la política de la $R P S P / P A J P H$ y/o de la OPS.

\section{REFERENCIAS}

1. GNUDS. Policy Brief: The Impact of Covid-19 on Latin America and the Caribbean. July 2020. [Internet]. [citado 9 Abril 2021]. Disponible en https://unsdg.un.org/es/resources/resumen-de-politicas-elimpacto-de-covid-19-en-america-latina-y-el-caribe.

2. Abdoul-Azize HT, El Gamil R. Social protection as a key tool in crisis management: learnt lessons from the COVID-19 pandemic. Glob Soc Welf 2020:1-10.

3. Human Rights Watch COVID-19 and Children's Rights. [Internet] New York: Human Rights Watch; 2021[citado 9 Abril 2021]. Disponible en: https:/ / www.hrw.org/news / 2020/04/09/ COVID-19-and-childrens-rights.

4. UNICEF. Impacto de la crisis por COVID-19 sobre los niños y niñas más vulnerables. [Internet]. Madrid: UNICEF;2021 [citado 9 abril 2021]. Disponible en: https://www.unicef.es/ impacto-covid19-infancia.

5. UNESCO. La educación en América Latina y el Caribe ante la COVID-19. [Internet]. Santiago de Chile: UNESCO; 2021[citado, 19 noviembre 2020]. Disponible en: https:/ / es.unesco.org/fieldoffice/ santiago/covid-19-education-alc/respuestas.

6. UNESCO. Marco para la reapertura de escuelas. [Internet]. [citado, 19 noviembre 2020]. Disponible en: https://es.unesco.org/sites/ default/files/marco_reapertura_escuelas_es.pdf

7. Naciones Unidas Derechos Humanos. Convención sobre los Derechos del Niño. [Internet]. Naciones Unidad 2021 [citado, 18 febrero 2021]. Disponible en: https://www.ohchr.org/sp/professionalinterest/ pages/crc.aspx

8. Garrido Gabriela, González Gabriel. ¿La pandemia de COVID-19 y las medidas de confinamiento aumentan el riesgo de violencia hacia niños/as y adolescentes? Arch. Pediatr. Urug. 2020 91(4): 194-195.

9. Singh S, Roy D, Sinha K, Parveen S, Sharma G, Joshi G. Impact of COVID-19 and lockdown on mental health of children and adolescents: A narrative review with recommendations. Psychiatry Research. 2020;293:113429. 
10. Reading R, Bissell S, Goldhagen J, Harwin J, Masson J, Moynihan S, Parton N, Pais MS, Thoburn J, Webb E. Promotion of children's rights and prevention of child maltreatment. Lancet. 2009 Jan 24;373(9660):332-43. doi: 10.1016/S0140-6736(08)61709-2. Epub 2008 Dec 4. PMID: 19056117

11. Engeli I, Rothmayr Allison C. Comparative policy studies. 1ra ed. Basingstoke: Palgrave Macmillan; 2014.

12. Bacchi C, Goodwin S. Poststructural policy analysis. New York, New York: Palgrave Macmillan; 2016.

13. Instituto de Evaluación Tecnológica en Salud - IETS-. Manual de Procesos Participativos. Bogotá D.C.; IETS; 2014: 43-45. Disponible en: http:/ / www.iets.org.co/Archivos/65/ManualParticipacion. pdf.

14. MSPS. Documentos Técnicos covid-19 Minsalud.gov.co. 2021 [Internet].Bogota:MSPS; [citado 11 Abril 2021]. Disponible en: https: / / www. minsalud.gov.co/salud/publica/PET/Paginas/Documentostecnicos-covid-19.aspx

15. El Peruano. Estado de Emergencia Principales Normas emitidas durante el Estado de Emergencia por el COVID-19 [Internet]. Lima: Diario Oficial El Peruano; 2021 [citado 11 abril 2021]. Disponible en: https://diariooficial.elperuano.pe/Normas/COVID19

16. Ministerio de Salud de Chile. Información Técnica COVID-19. [Internet]. Santiago: Ministerio de Salud de Chile. [citado 11 agosto 2021]. Disponible en: https://www.minsal.cl/nuevo-coronavirus2019-ncov/informe-tecnico/

17. Quinn Patton M. Qualitative research \& evaluation methods. Integrating Theory and Practice. 4ta ed. Los Angeles: SAGE; 2015.

18. Abbey EJ, Khalifa BAA, Oduwole MO, et al. The global health security index is not predictive of coronavirus pandemic responses among organization for economic cooperation and development countries. PLoS One 2020;15:e0239398

19. Renzaho AMN. The Need for the Right Socio-Economic and Cultural Fit in the COVID-19 Response in Sub-Saharan Africa: Examining Demographic, Economic Political, Health, and Socio-Cultural Differentials in COVID-19 Morbidity and Mortality..Int J Environ Res Public Health. 2020 May 15;17(10):3445. doi: 10.3390/ijerph17103445. PMID: 32429123

20. Banco Interamericano de Desarrollo. El alto costo del COVID-19 para los Niños. Estrategias para mitigar su impacto en América Latina y el Caribe. 2020. [Internet]. [citado, 4 septiembre 2020]. Disponible en: https:/ / publications.iadb.org/es/el-alto-costo-del-covid-19-paralos-ninos-estrategias-para-mitigar-su-impacto-en-america-latinay-caribe.

21. Johnston RM, Mohammed A, van der Linden C. Evidence of exacerbated gender inequality in child care obligations in Canada and Australia during the COVID-19 pandemic. Pol \& Gen 2020; 16:1131-41.

22. UNICEF. Repensar el tiempo de pantalla en el tiempo de COVID-19. [Internet]. [citado, 4 Septiembre 2020]. https://www. unicef.org/colombia/historias/repensar-el-tiempo-de-pantalla-en-eltiempo-de-covid-19

23. Engzell P, Frey A, Verhagen MD. Learning loss due to school closures during the COVID-19 pandemic. Proc Natl Acad Sci U S A 2021;118:e2022376118.

24. UNICEF insta al compromiso por una crianza sin violencia Unicef. org. 2021 [Internet]. [citado 11 abril 2021]. Disponible en: https:// www.unicef.org/colombia/comunicados-prensa / unicef-instaal-compromiso-por-una-crianza-sin-violencia
25. Roth Deubel, André-Nöel; Pinzón Segura, María Camila, MosqueraPedreros N, Cajamarca L, Méndez D, Aragón-Joya (2021) Guía para la realización de Cabildos Ciudadanos en Colombia. Bogotá: Universidad Nacional de Colombia. ISBN 978-958-794-496-9

26. Hart, Roger A. (1993). La participación de los niños: de una participación simbólica a una participación auténtica, en Ensayos Innocenti no4. UNICEF.

27. Ministerio de Salud y Protección Social de Colombia. (2019). Política de Participación Social en Salud PPSS. Resolución 2063 de 2017. [Internet] Bogota: Ministerio de Salud y Protección Social de Colombia; [citado, 4 septiembre 2020]. https:/ / www.minsalud.gov. $\mathrm{co} /$ sites/rid/Lists/BibliotecaDigital/RIDE/DE/GT/politica-ppssresolucion-2063-de-2017-cartilla.pdf

28. Save the Children. Estudio conceptual rápido de C-19 en poblaciones de alta densidad y pobres en recursos. 2021 [Internet]. [citado 11 abril 2021]. Disponible en: https://savethechildren.org.co/ estudio-conceptual-rapido-de-c19-poblaciones-de-alta-densidady-pobres-en-recursos-2/

29. CEPAL (2020). Protección social para familias con niños, niñas y adolescentes en América Latina y el Caribe Un imperativo frente a los impactos del COVID-19 [Internet]. [citado, 4 de septiembre 2020]. Disponible en: https://repositorio.cepal.org/bitstream/ handle/11362/46489/1/S2000745_es.pdf

30. Pinzón-Segura M. Políticas Públicas, Implementación y Emociones: Hacia un Enfoque Alternativo de Análisis. Caso de estudio: Política Pública de Salud para Niñas, Niños y Adolescentes con Cáncer en Bogotá - Colombia [Doctorado interfacultades en Salud Pública]. Universidad Nacional de Colombia; 2016.

31. Pinzón Segura, María Camila; Ballesteros Cabrera, Magnolia de Pilar; Prieto Villate, Silvia; Díaz Romero, Orlando. 2020. LOS REGALOS DE MARTINA. Centro Editorial Facultad de Medicina Universidad Nacional de Colombia. 2020. ISBN 978-958-794-129-6

32. Almeida-Filho N, Silva Paim J. La crisis de la salud pública y el movimiento de salud colectiva en Latinoamérica. Cuadernos Médico Sociales. 1999; 75:5-30.

33. Breilh Jaime. La Determinación Social de la salud “Una Perspectiva Emancipadora de la Investigación y Acción, Basada en la Determinación Social de la Salud". Ponencia, Taller Latinoamericano de Determinantes Sociales de la Salud, 29 de septiembre al 2 de octubre, México D.F: Alames: 2008.

34. Uzcátegui JL et al. Sociedad, política y salud en tiempos de pandemia. - la ed - Ciudad Autónoma de Buenos Aires: CLACSO Xochimilco: Universidad Autónoma Metropolitana; Xalapa: Universidad Veracruzana; Cochabamba: Universidad Mayor de San Simón; Valparaíso : Universidad de Valparaíso, 2021. [Internet]. [citado, 22 agosto 2021]. Disponible en: http:/ / biblioteca.clacso.edu. ar/clacso/gt/20210312065632/America-Latina-Sociedad-politicay-salud.pdf

35. Breilh J. Epidemiología crítica: ciencia emancipadora e interculturalidad. Buenos Aires: Lugar Editorial; 2003.

Manuscrito recibido el 3 de julio del 2021. Aceptado para su publicación, tras revisión, el 1 de octubre del 2021. 


\section{Response to the COVID-19 Pandemic in Chile, Colombia, and Peru from a children's rights perspective}

ABSTRACT Objective. Describe the strategies established by Chile, Colombia, and Peru during the first year of the COVID-19 pandemic and compare them from a children's rights perspective.

Methods. A qualitative study with comparative analysis of public policies was conducted around seven categories constructed by the Latin American Chapter of the International Society for Social Pediatrics and Child Health, based on the Convention on the Rights of the Child. Country documents were selected based on convenience sampling and were analyzed in deliberative dialogues.

Results. 173 documents from the three countries were reviewed. There was convergence around: prioritization of prevention of community transmission of the virus over promotion of the exercise of children's rights; lack of participation of children and adolescents in the process of developing public policies; and lack of progress in the recognition and protection of the exercise of children's rights overall. There were no major divergences beyond identified inequality gaps grounded in the reality of each country.

Conclusion. The pandemic has affected the functioning of the economic, social, health, education, environmental, and governance systems in these three countries. While this study shows progress in the inclusion of the children's rights approach in formulated policies, the recognition of children and adolescents as holders of social and political rights could allow the construction of collective alternatives that guarantee health and well-being for all people throughout the life course.

Keywords COVID-19; child advocacy; health policy; public policy; child care; child health; Chile; Colombia, Peru.

\section{Resposta à pandemia de COVID-19 no Chile, Colômbia e Peru com foco nos direitos da criança}

RESUMO

Objetivo. Descrever as estratégias que foram utilizadas por Chile, Colômbia e Peru durante o primeiro ano da pandemia de COVID-19 e compará-las a partir da perspectiva dos direitos da criança.

Métodos. Realizou-se um estudo qualitativo de análise comparativa de políticas públicas, tomando como eixo sete categorias construídas pelo Capítulo Latino-americano da International Society for Social Pediatrics \& Child Health a partir da Convenção sobre os Direitos da Criança (CDC). A seleção de documentos dos países foi feita por conveniência, e sua análise ocorreu mediante diálogos deliberativos.

Resultados. Foram revisados 173 documentos dos três países. A prioridade da prevenção da transmissão comunitária do vírus destaca-se por sua convergência, em detrimento da promoção do exercício dos direitos da criança, da falta de participação de crianças e adolescentes no processo de formulação de políticas públicas e da falta de progresso no reconhecimento e proteção da efetivação de todos os seus direitos. Não houve grandes divergências além das lacunas de desigualdade identificadas com base na realidade de cada país. Conclusões. A pandemia afetou o funcionamento dos sistemas econômico, social, de saúde, educação, meio ambiente e governança desses três países. Embora este estudo mostre avanços na inclusão da perspectiva dos direitos da criança e do adolescente nas políticas formuladas, a compreensão deles como sujeitos sociais e políticos titulares de direitos pode permitir a construção de alternativas coletivas que garantam saúde e bem-estar a todas as pessoas ao longo do curso da vida.

Palavras-chave COVID-19; defesa da criança e do adolescente; política pública; cuidado da criança; saúde da criança; Chile; Colombia, Perú. 